\title{
Families of Vortex Solitons in Periodic Media
}

\author{
Jiandong Wang, Jianke Yang \\ Department of Mathematics and Statistics, University of Vermont, Burlington, VT 05401, USA
}

\begin{abstract}
Various families of charge-one vortex solitons in two-dimensional periodic media are reported. These vortices reside either in the semi-infinite gap or higher band gaps of the media. For both Kerr and saturable nonlinearities (either focusing or defocusing), infinite vortex families are found. All these families do not bifurcate from Bloch bands; rather, they turn around before reaching edges of Bloch bands. It is further revealed that vortices with drastically different topological shapes can belong to the same vortex family, which is quite surprising.
\end{abstract}

PACS numbers: 42.65.Tg, 05.45.Yv

\section{INTRODUCTION}

Nonlinear wave propagation in periodic media is an important phenomena, and it arises in a wide array of physical settings such as photonic-crystal fibers [1, 2], photorefractive crystals imprinted with a photonic lattice [3, 4], and Bose-Einstein condensates loaded in an optical lattice [5, 6, 7]. The interplay between nonlinearity and periodicity results in a lot of new physical effects of the wave propagation. For instance, in a periodic media, solitons can exist not only under focusing nonlinearity, but also under defocusing nonlinearity [8, 9, 10]. A distinctive feature of the periodic media is that it can support a wide variety of solitons residing in different band gaps. Examples include fundamental solitons [9, 11, 12, 13, 14, 15, 16, 17], dipole solitons [18, 19], vortex solitons [13, 20, 21, 22, 23], reduced-symmetry solitons [24], higher-band vortex solitons [25, 26, 27], embedded-soliton trains [28], and so on - many of which have been experimentally observed. Solitons in Besselring lattices have been reported too [29, 30]. In an effort to classify solitons in two-dimensional (2D) periodic media, Shi and Yang 31] studied low-amplitude solitons near Bloch-band edges. These solitons are Bloch waves modulated by slowly-varying envelope functions. Using asymptotic techniques, they derived envelope equations, based on which they successfully classified all soliton families bifurcating from Bloch-band edges. Examples include fundamental solitons, reduced-symmetry solitons, dipole-array solitons, and many others. However, a peculiar question arose on vortex solitons. According to the envelope equations, those vortex solitons should also bifurcate from Bloch-band edges. However, numerical results suggest that they disappear before reaching band edges [32, 33]. This paradox has not been resolved yet, and it clouds over the vortex-soliton research in periodic media.

In this paper, we comprehensively investigate chargeone vortex solitons residing in various band gaps of a $2 \mathrm{D}$ periodic media, and resolve the above paradox on this subject. For both the Kerr (cubic) and saturable nonlinearities, we find that there exist infinite families of on-site and off-site vortex solitons in the semi-infinite gap (for focusing nonlinearity) and in the first gap (for defocusing nonlinearity). We further show that all these vortex families do not bifurcate from edges of Bloch bands. Rather, before reaching band edges, they turn back and move into band gaps again. Within each vortex family, shapes of vortex solitons undergo drastic changes as the propagation constant varies. As a result, vortices with very different topological structures can actually belong to the same family - a phenomenon which has never been reported before. These findings significantly deepen our understanding on vortex solitons in general periodic media.

\section{VORTEX SOLITONS UNDER KERR NONLINEARITY}

We first consider vortex solitons in 2D periodic media under Kerr nonlinearity. The mathematical model for this situation is

$$
i U_{z}+U_{x x}+U_{y y}+V(x, y) U+\sigma|U|^{2} U=0,
$$

where $U$ is a complex function, $\sigma$ takes the value of 1 for focusing nonlinearity and -1 for defocusing nonlinearity, and $V(x, y)$ is a $2 \mathrm{D}$ periodic potential. This equation arises naturally in Bose-Einstein condensates loaded in an optical lattice [5, 6, 7] as well as nonlinear light propagation in laser-written waveguides [34] and photonic crystal fibers with weak transverse index variation. Without loss of generality, we take the periodic potential $V(x, y)$ as

$$
V(x, y)=-V_{0}\left(\sin ^{2} x+\sin ^{2} y\right),
$$

and take $V_{0}=6$ in all our calculatioins.

Vortex solitons in Eq. (1) are sought in the form

$$
U(x, y ; z)=u(x, y) \exp (-i \mu z),
$$

where $\mu$ is the propagation constant, and $u(x, y)$ is a complex-valued localized function which satisfies the equation

$$
u_{x x}+u_{y y}+[\mu+V(x, y)] u+\sigma|u|^{2} u=0 .
$$

We will determine these solutions numerically using the modified squared-operator iteration method and the power-conserving squared-operator method developed in [35]. 


\section{A. The Case of Focusing Kerr Nonlinearity}

First, we study vortex solitons supported by the focusing Kerr nonlinearity, i.e. $\sigma=1$ in Eq. (4). Two types of vortex solitons - on-site and off-site ones, will be sought. On-site vortices are centered on a lattice site (lattice peak), while off-site vortices are centered between lattice sites (lattice minimum) [13, 20, 21, 22]. For each type of vortices, various families are found in the semiinfinite gap. The power diagrams of the first two families of on-site and off-site vortex solitons are shown in Fig. 1] (a, b) respectively. Here the power is defined by $P=\int_{-\infty}^{\infty} \int_{-\infty}^{\infty}|u|^{2} d x d y$. A distinctive feature about these power curves is that each curve has a slanted Ushape. At each propagation constant $\mu$ away from the band edge, one can find two vortex solitons with different powers within each family. Another feature about these curves is that none of them reaches the edge of the (first) Bloch band. This means that none of these vortex families bifurcates from Bloch bands. These features are in stark contrast with those for soliton families reported in [31], which do bifurcate from Bloch bands (in the low-amplitude limit), and each propagation constant only corresponds to a single soliton solution within each family. To find out what types of vortex solitons are contained in each family, we display them at four representative locations of each power curve in Fig. 2 (on-site) and Fig. 3 (off-site). First we examine the first on-site vortex family whose power curve is the solid (blue) one in Fig. 1(a). At four marked positions 'a-d' on this curve, the corresponding vortex profiles are displayed in Fig. 2(a-d) respectively. The phase structures of all these vortices are similar to that of the ring vortex soliton in bulk media and shown in the inset of Fig. 2(a). Winding around the center of the vortex, the phase increases by $2 \pi$, thus these vortices have charge one. The intensity distributions of these vortices, on the other hand, differ significantly at various locations of the power curve. On the lower branch of the power curve, when $\mu$ is far away from the Bloch band, the vortex soliton consists of four main humps residing in the four lattice sites closest to the center site and forming a diamond configuration [see Fig. 2 (a)]. Hence this is the familiar on-site vortex soliton which has been reported before [20, 22, 36]. When $\mu$ increases toward the band edge along the lower branch, the vortex's power as well as its peak intensity decreases, and the vortex starts to develop tails on the outside of its four main humps [see Fig. 2 (b)]. As $\mu$ further increases beyond a cut-off value $\mu_{c}^{(1)}=4.107$, which is close to but below the band edge $\mu_{\text {edge }}=4.126$, vortices in this family can not be found anymore. This phenomenon was first reported by one of the authors in [32], and it was puzzling at the time since we did not expect a solution branch terminating abruptly away from edges of Bloch bands. This puzzle is now resolved by Fig. 1(a). What happens is that when $\mu$ reaches the cut-off value $\mu_{c}^{(1)}$, the power curve turns around and enters the upper branch of the same family [see Fig. 1(a)]. When passing through the turn-around, the vortex undergoes rapid shape changes and evolves into eight main humps in a square configuration surrounding the central lattice site. Tails at the outside of this square are significant too. As $\mu$ moves away from the Bloch band along the upper branch, the tails gradually fade away, and the eight main humps intensity totally dominate the vortex [see Fig. 2 (d)]. Thus, in this first family of on-site vortex solitons, the vortex undergoes dramatic shape changes from a four-humped diamond-like structure into an eighthumped square structure. This is quite unexpected and was not known before.

Regarding the second on-site family, vortex solitons at four representative positions 'e-h' of the power curve are displayed in Fig. 2 (e-h). The phase structures of these vortices are similar to those in the first family [see inset of Fig. 2(e)], i.e. these vortices also have charge one. But the intensity patterns in this second family are quite different from those in the first family. Specifically, in the lower branch of the second family, away from the Bloch band, the vortex contains eight main humps in a larger-diamond configuration [see Fig. 2(e)]. As $\mu$ moves continuously from the lower branch to the upper one, the vortex continuously evolves from this eight-humped diamond configuration to a sixteen-humped configuration resembling the surrounded wall of a castle [see Fig. 2(eh)]. This dramatic shape change within the same solution family occurs for all vortex families, and it is one of the main findings of this article.

For off-site vortex solitons in the semi-infinite gap, various solution families are found as well. In the first off-site family, representative solution profiles are displayed in Fig. 3 (a-d). These vortices also have a simple $2 \pi$ phase winding structure around the vortex center, thus having charge one [see inset in Fig. 3(a)]. Regarding their intensity patterns, when $\mu$ is far away from the Bloch band on the lower branch, the vortex has four main humps occupying four adjacent lattice sites in a compact square configuration. This is the familiar off-site vortex soliton reported in [13, 21, 22, 32]. As $\mu$ moves from the lower branch to the upper one, the vortex changes from the four-humped square configuration to a twelve-humped compact cross configuration [see Fig. 3(a-d)]. In the second off-site family, lower-branch vortex solitons away from the Bloch band have eight main humps arranged in an octagon configuration [see Fig. 3(e)]. As $\mu$ moves to the upper branch, however, the vortex becomes a square with twelve main humps on its perimeter [see Fig. 3(h)]. Notice from Fig. 1 (b) that the upper branches of these two off-site families have almost the same power for the same propagation constant. This is because on these two upper branches, vortices of both families have the same number of main humps, and nearby humps are all aligned along the lattice direction and separated by one lattice spacing [see Fig. 3(d,h)]. One may recall that for onsite vortices, the upper branch of the first family and the lower branch of the second family also have the same 
(a)

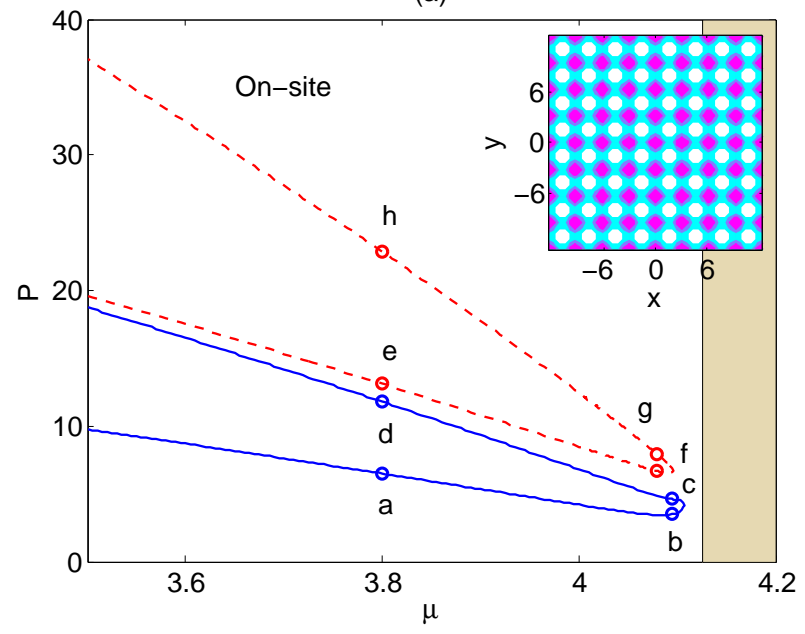

(b)

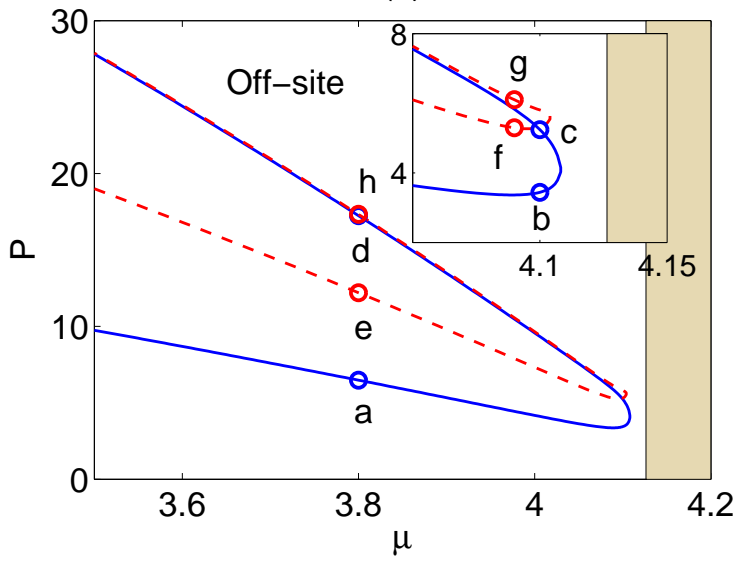

FIG. 1: (Color online) Power diagrams of the first two families of on-site (a) and off-site (b) vortex solitons in the semi-infinite gap under focusing Kerr nonlinearity. The inset in (a) shows the 2D square lattice of Eq. (2); the inset in (b) zooms in on the graph near the band edge. Vortex profiles at the marked points are shown in Fig. 2 (on-site) and Fig. 3 (off-site) respectively. Shaded: the first Bloch band.

(a)

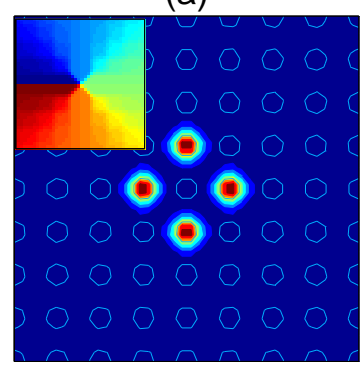

(e)

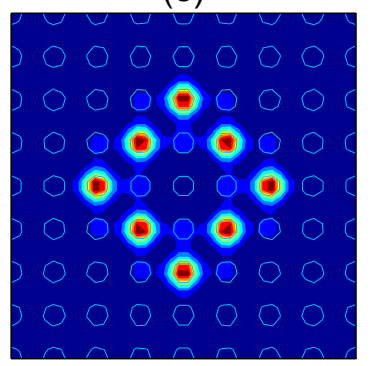

(b)

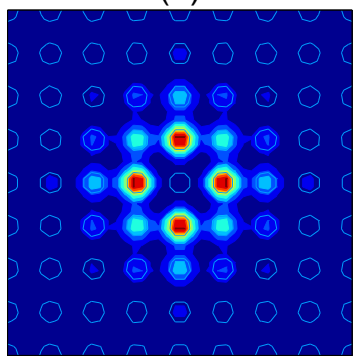

(f)

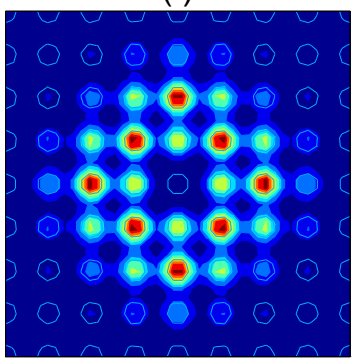

(c)

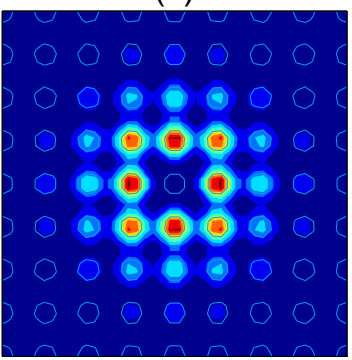

(g)

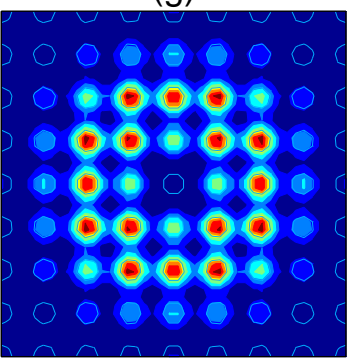

(d)

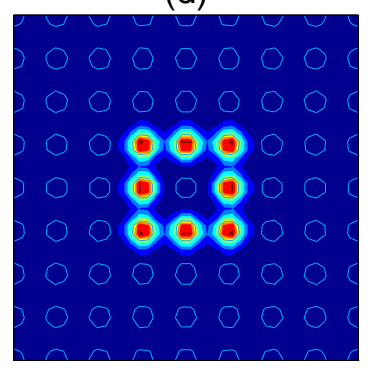

(h)

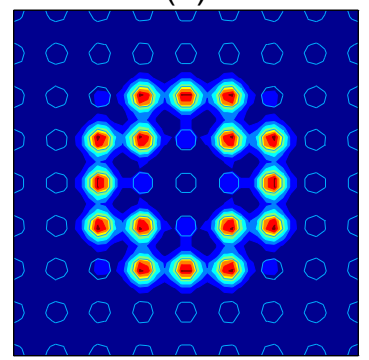

FIG. 2: (Color online) Vortex profiles $(|u|)$ in the first (top) and second (bottom) on-site solution families under focusing Kerr nonlinearity at marked points in Fig. 1(a). The inset in (a) is the typical phase structure of all these vortices. The background circles represent the lattice sites (with high $V$ values), as in Figs. 3, 5 and 6 as well.

number of humps [see Fig. 2(d, e)]. But adjacent humps in the latter case are aligned along diagonal directions of the lattice and have larger spatial separations than in the former case. This caused noticeable power differences between these two branches as can be seen in Fig. 1 (a).

From Fig. 1, we see that both on-site and off-site vortices can exist close to the Bloch band [see points 'c, g' in Fig. 1(a) for example]. We find that such vortices near the Bloch band have lower amplitudes. For in- stance, the vortex at point ' $c$ ' in Fig. 1(a) has amplitude 0.55 , and the one at point 'g' has amplitude 0.48. Comparatively, vortex amplitudes far away from the Bloch band are much higher. Additionally, vortices close to the Bloch band have long tails, and they resemble the Bloch wave of the band edge modulated by a ring-vortex envelope [see Fig. 2(c, g) for instance]. This seems to suggest that vortex solitons near Bloch bands can be treated analytically by asymptotic methods. However, 
(a)

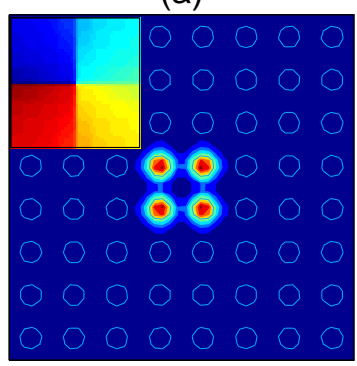

(e)

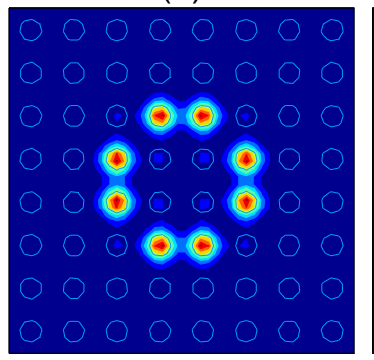

(b)

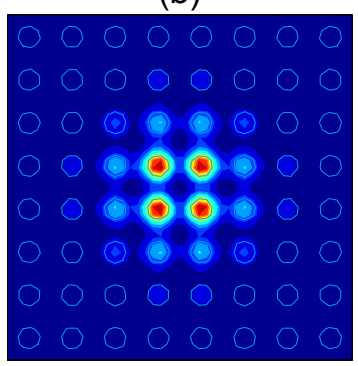

(f)

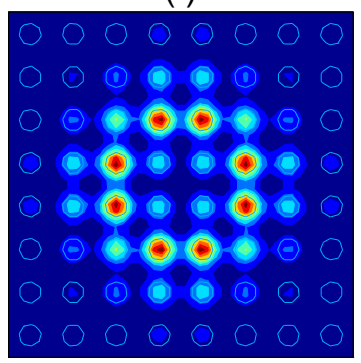

(c)

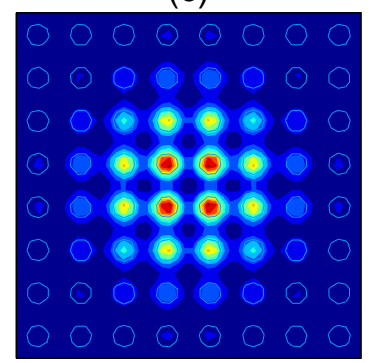

(g)

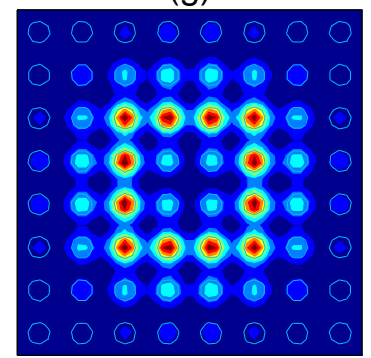

(d)

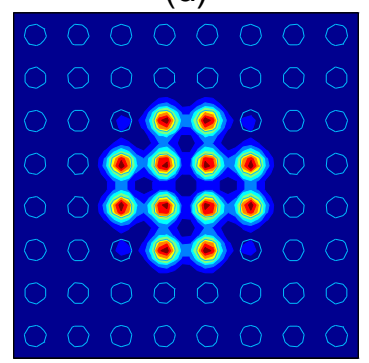

(h)

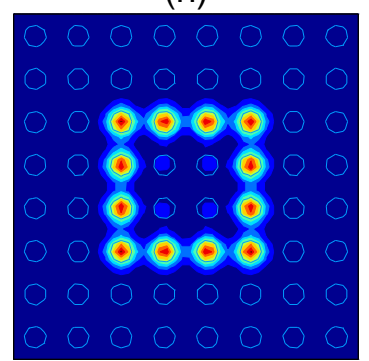

FIG. 3: (Color online) Vortex profiles $(|u|)$ in the first (top) and second (bottom) off-site solution families under focusing Kerr nonlinearity at marked points in Fig. 1 (b). The inset in (a) is the typical phase structure of all these vortices.

such asymptotic analysis encounters subtle problems for vortex solitons, as we will explain below. Low-amplitude Bloch-wave packets in Eq. (11) have been analyzed in 31]. Near the band edge $\mu_{e d g e}$ in Fig. 1 and under focusing nonlinearity, the leading-order asymptotic solution is $u(x, y)=\epsilon A(X, Y) p(x, y), \mu=\mu_{e d g e}-\epsilon^{2}$. Here, $p(x, y)$ is the Bloch wave at the band edge $\mu_{\text {edge }}$ (with $\Gamma$-point symmetry), $X=\epsilon x, Y=\epsilon y, \epsilon \ll 1$, and the envelope function $A(X, Y)$ satisfies the equation

$$
D_{1}\left(A_{X X}+A_{Y Y}\right)-A+\alpha_{0}|A|^{2} A=0,
$$

where $D_{1}>0$ is the second-order dispersion coefficient at the band edge, and $\alpha_{0}>0$ is a constant. The envelope equation (5) admits a ring-vortex solution $A=f(R) e^{i \Theta}$, where $(R, \Theta)$ is the polar coordinates of the $(X, Y)$ plane. Due to some additional constraints on the solution $u(x, y)$, this ring envelope can only be centered at certain locations at or between lattice sites [31]. Centering the ring-vortex envelope at a lattice site, the corresponding solution $u(x, y)$ would then be a low-amplitude on-site vortex soliton which resembles the ones such as Fig. 2 (c, g). Centering the ring-vortex envelope between a lattice site, we would get a low-amplitude off-site vortex soliton which resembles the ones in Fig. 3 (c, f). However, a contradiction between this asymptotic analysis and numerical results is that, according to the asymptotic analysis, these vortices should exist continuously as $\mu$ approaches the band edge $\mu_{\text {edge }}$ (i.e. $\epsilon \rightarrow 0$ ), but the numerical results in Fig. 1 indicate that true vortex solitons actually do not approach the band edge for each vortex family. Apparently, the asymptotic analysis above is not entirely correct for vortex solitons, and it must be revised in order to explain the true vortex behaviors in Figs. 1-3. How this can be done is still unclear yet. Note that these vortex behaviors are similar to multi-packet solitary waves in the fifth-order Korteweg de Vries equation [37], so the analytical treatment of 37] might be useful for the present problem. This remains to be seen.

Fig. 1 just shows the first two families of on-site and off-site vortex solitons. We have also found other vortex families in Eq. (4) whose power curves have similar slanted U-shapes and are higher than those in Fig. 1. Vortices in these higher families contain more intensity humps which are located further away from the center of the vortex, but their phase structures remain similar to those of the first two families (i.e. they all have charge one). It can be inferred that infinite families of such vortex solitons exist in the semi-infinite gap, and all of them do not bifurcate from the edge of the first Bloch band. Within each higher family, vortex shapes also undergo drastic changes as $\mu$ moves from the lower branch to the upper one.

The vortex solitons studied above are symmetric about the vortex center. Asymmetric vortices also exist in a square lattice [23]. We find that the power curves of asymmetric vortices also have slanted U-shapes as in Fig. 1 , and they do not bifurcate from Bloch bands either. We also find that asymmetric vortices reported in [23] belong to the lower branches of these power curves. As $\mu$ moves to the upper branches, shapes of asymmetric vortices become very different and more complex. 
(a)

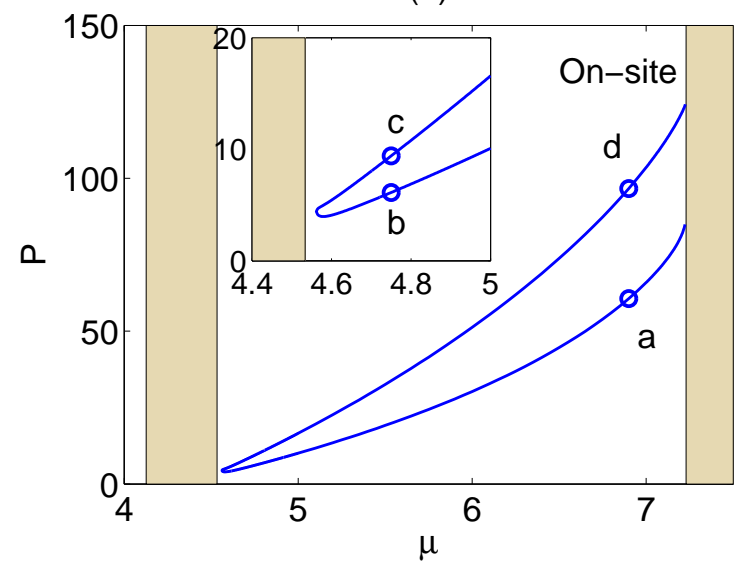

(b)

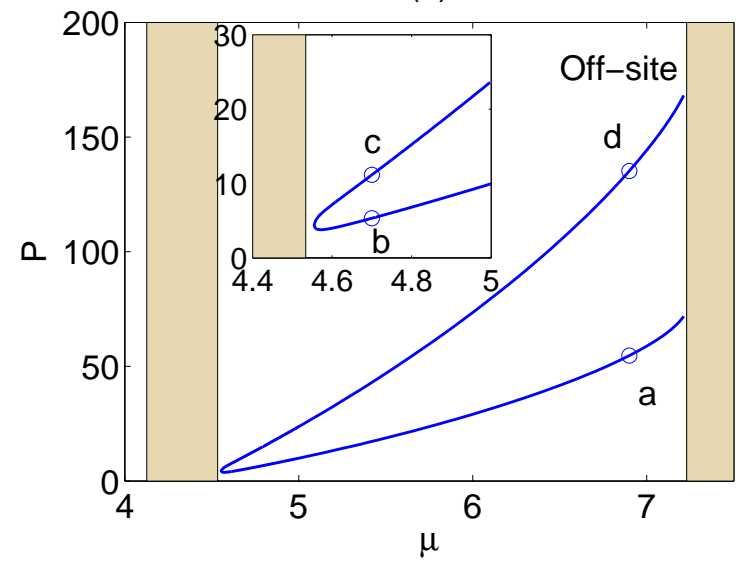

FIG. 4: Power diagrams of the first family of on-site (a) and off-site (b) vortex solitons in the first band gap under defocusing Kerr nonlinearity. The insets zoom in on the graphs near the band edge. Vortex profiles at the marked points are shown in Fig. [5 (on-site) and Fig. 6] (off-site) respectively. Shaded: the first two Bloch bands.

(a)
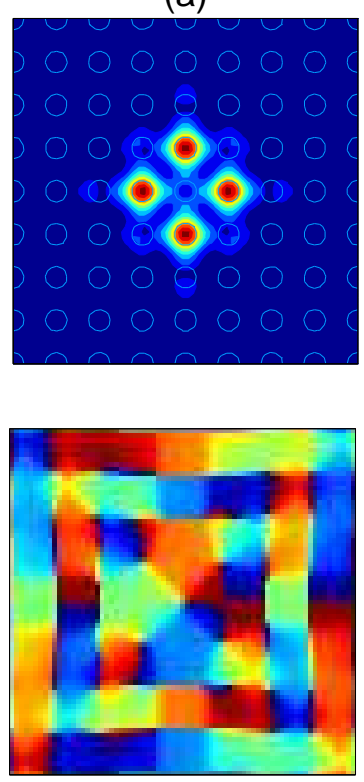

(b)
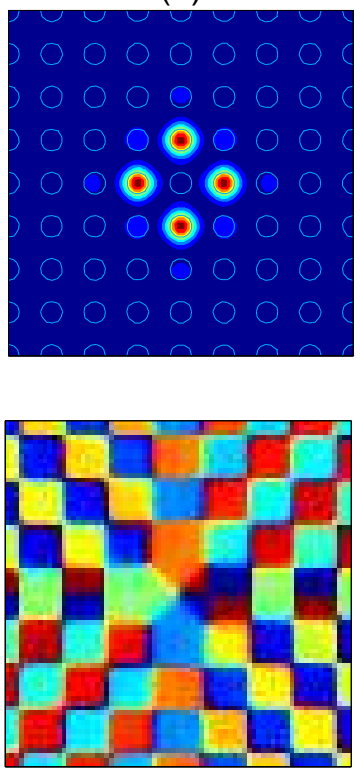

(c)
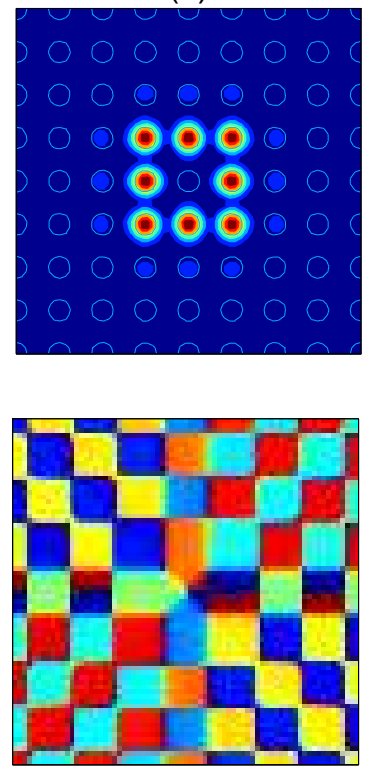

(d)
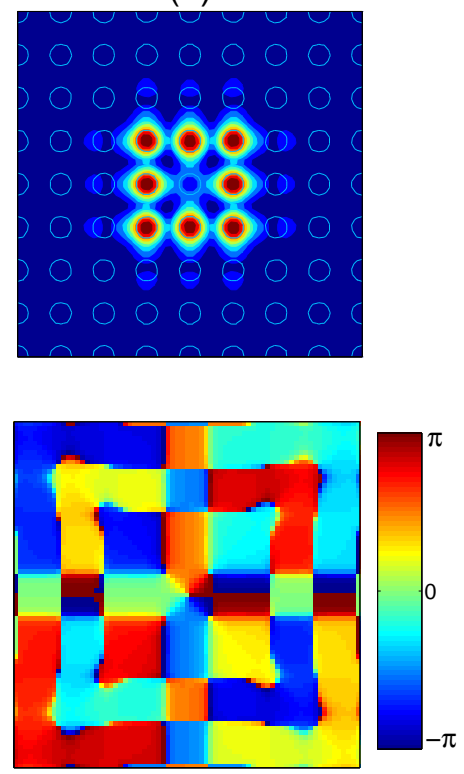

FIG. 5: (Color online) On-site gap vortex profiles ( $|u|$, top row) and phase structures (bottom row) corresponding to the marked points in Fig. 4 (a) under defocusing Kerr nonlinearity. Bottom right: color bar of the phase figures.

\section{B. The Case of Defocusing Kerr Nonlinearity}

In this subsection, we examine vortex solitons under defocusing Kerr nonlinearity, i.e. $\sigma=-1$ in Eq. (4). These vortices exist as infinite families in the first band gap. Fig. 4 shows the power curves of the first on-site and off-site gap-vortex families. It is seen that these power curves also have a U-shape, but slanted in an opposite direction from the focusing case (see Fig. 1). Spatial profiles and phase structures of these gap vortices at the marked points in Fig. 4 are displayed in Fig. 5 (on-site) and Fig. 6] (off-site). We see that the shapes of these firstfamily defocusing gap vortices $(|u|)$ resemble those of the first-family focusing vortices. Specifically, for the first onsite defocusing family, as $\mu$ moves from the lower branch to the upper one, the shape of gap vortices changes from a four-humped diamond configuration to an eight-humped square configuration (see Fig. [5), analogous to the focusing case (see Fig. 2). For the first off-site defocusing family, as $\mu$ moves from the lower branch to the upper one, gap vortices change from a four-humped square configuration to a twelve-humped cross configuration (see Fig. 6), also analogous to the focusing case (see Fig. 3). The most important difference between defocusing vortices and focusing vortices lies in their phase structures. The phase fields of focusing vortices have a simple $2 \pi$ - 
(a)
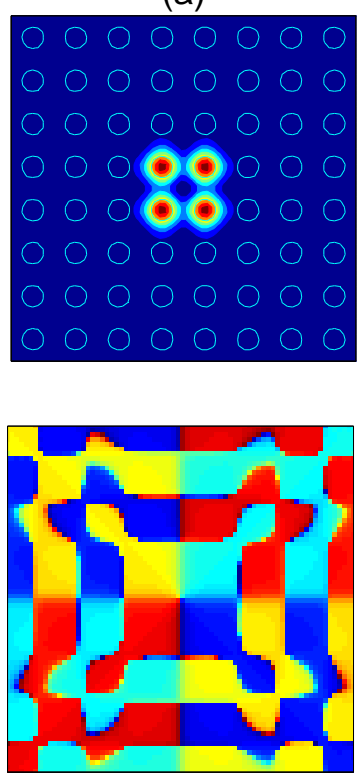

(b)
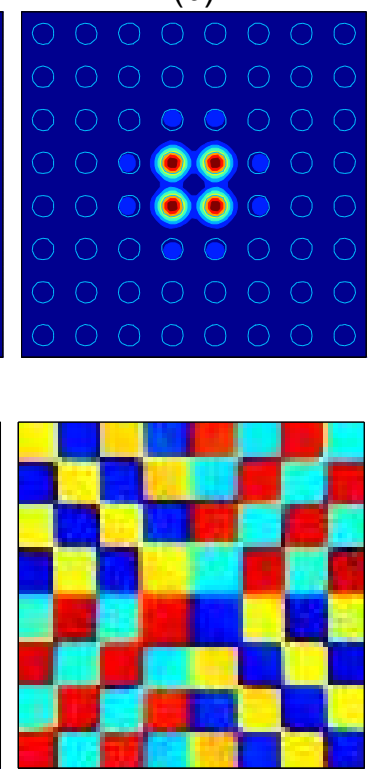

(c)
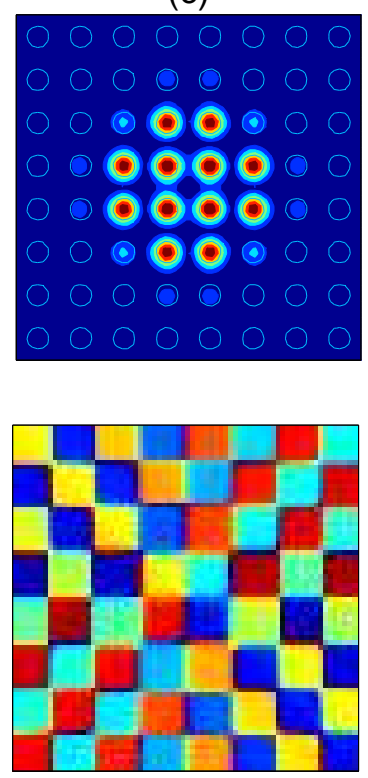

(d)
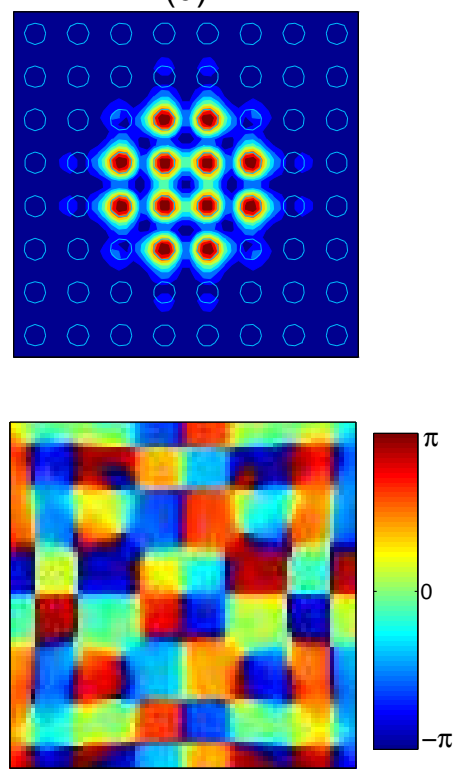

FIG. 6: (Color online) Off-site gap vortex soliton profiles $(|u|$, top row) and phase structures (bottom row) corresponding to the marked points in Fig. 4 (b) under defocusing Kerr nonlinearity. Bottom right: color bar of the phase figures.

winding structure like in ring vortices of bulk media [see insets in Figs. 2(a) and 3(a)]. However, phase structures of defocusing gap vortices are much more complex (see Figs. 5 and 6). In the central region of gap vortices, the phase field has a simple $2 \pi$-winding structure around the vortex center. But in the outer region, vortex phases at adjacent lattice sites sometimes have $\pi$ difference [see Figs. [5(b,c) and 6(b,c)], but some other times do not [see Figs. 5(d) and 6(a)]. These complicated phase structures make the concept of topological charges ambiguous and not well defined for gap vortices. Note that gap vortices reported earlier in [25, 33] correspond to the lower branches of our gap vortices in Fig. 4]

From Fig. 4 we see that gap vortices can exist quite close to the right edge of the first Bloch band. At this band edge, the second-order dispersion coefficient $D_{1}$ is negative. Thus according to the envelope equation for this band edge, which is similar to Eq. (5) with only the sign of the $A$ term reversed, ring-vortex solutions $A=f(R) e^{i \Theta}$ exist under defocusing nonlinearity (where $\alpha_{0}<0$ ). Hence for $\mu$ close to this band edge, one may construct the analytical gap-vortex solution $u(x, y)=\epsilon A(X, Y) p(x, y)$, where $p(x, y)$ is the Bloch wave at this right edge (with $M$-point symmetry). This analytical gap-vortex solution qualitatively reproduces the intensity distribution, and more importantly, the intricate phase structure, of the true solution near the right edge of the first band [see Figs. 5(b) and 6(b) for instance]. In particular, it explains the simple $2 \pi$ winding of the phase near the vortex center, and the $\pi$ phase difference between lattice sites in the far field (due to the $M$-point symmetry of the Bloch wave at this band edge). Of course, this analytical gap-vortex solution can not explain why true gap vortices exist as infinite families, and each family does not continuously reach the band edge - the same difficulty we have seen with the focusing nonlinearity in the previous subsection.

\section{VORTEX SOLITONS UNDER SATURABLE NONLINEARITY}

In photorefractive crystals where many lattice-soliton experiments were performed in recent years, the nonlinearity is saturable [38]. Even though wave phenomena under saturable and Kerr nonlinearities have many features in common, significant differences exist as well. In this section, we examine vortex solitons under saturable nonlinearity.

A simple theoretical model for solitons in photorefractive crystals imprinted with a photonic lattice is [36, 38]

$$
u_{x x}+u_{y y}-\frac{E_{0}}{1+I(x, y)+|u|^{2}} u=-\mu u
$$

where $E_{0}$ is the applied dc field,

$$
I(x, y)=I_{0} \cos ^{2}(x) \cos ^{2}(y)
$$

is a periodic square-lattice function, $I_{0}$ is the peak intensity of this lattice, and $\mu$ is the propagation constant. Here all variables have been non-dimensionalized [36]. When the soliton amplitude is low $(|u| \ll 1)$, the saturable nonlinearity becomes the same as the Kerr nonlinearity. Therefore, we can expect that near Bloch bands, the vortex behavior under saturable nonlinearity should be similar to that under Kerr nonlinearity. In particular, they can not bifurcate from Bloch bands either. 
Below we numerically investigate vortex solitons in the semi-infinite gap in Eq. (6) under focusing nonlinearlty $\left(E_{0}>0\right)$. Without loss of generality, we choose parameters $I_{0}=3$ and $E_{0}=6$. Vortex solitons in the first band gap under defocusing saturable nonlinearity will be briefly described in the end of this section.

First, we examine on-site vortex solitons under focusing saturable nonlinearity. An infinite number of such vortex families exist in the semi-infinite gap, and one of them is reported below. The power curve of this family is displayed in Fig. 7. We see that this curve is quite complicated. It has a number of turning points (twists), and winds up and down in an unexpected way (a somewhat similar power curve was reported for 1D lattice soltons under cubic-quintic nonlinearities in [39]). All vortices in this family have simple $2 \pi$ phase winding structures, thus having charge one [see inset of Fig. $8(a)]$. Their intensity structures along the power curve are displayed in Fig. 8. It is seen that far from the band edge (point ' $a$ ' in Fig. 7), the power is very high, and the vortex has a ring profile [see Fig. $8(\mathrm{a})$ ] . This ring profile can be easily understood since the vortex here has high intensities and dominates over the lattice, thus it effectively becomes a ring vortex in a lattice-free (bulk) media. As the power and intensity decrease (when $\mu$ increases), the lattice effect starts to appear, and the vortex reshapes itself by distributing its main power to the lattice sites. This leads to a four-humped on-site vortex as seen in Fig. $8(\mathrm{~b}, \mathrm{c})$. This vortex has been theoretically analyzed in [36] and experimentally observed in [22]. This reshaping process, however, is a delicate one as is evidenced by the two turning points near ' $\mathrm{b}$ ' in the power curve of Fig. 7 (a). As the power curve gets close to the first band (see points 'c, d'), the vortex starts to spread out, and its amplitude becomes low. Vortices here closely resemble those of the first on-site Kerr family near the first band [see Fig. $2(b, c)]$. Therefore, the power curve of saturable vortices can not reach the first band either, and has to turn around before reaching the band edge. After the turning point, the power starts to increase (as $\mu$ moves away from the Bloch band), and the vortex reshapes itself again. This time, after a complex reshaping process, the eight-humped square vortex at point 'd' becomes a four-humped square vortex with its humps residing near the four diagonal lattice sites around the vortex center [see Fig. $8(\mathrm{e}, \mathrm{f})$ ]. Strangely, in this reshaping process, the power curve turns around again at $\mu=1.832$ [between ' $e$ ' and ' $f$ ' in Fig. 7 (a)], and starts to decrease toward the first band [see ' $\mathrm{g}$ ' in Fig. 7 (b)]. Near the first band, the power curve turns around again, and moves back into the semi-infinite gap [see inset in Fig. 7(b)]. Near the turning point, the vortex spreads out again [see Fig. 8 $(h, i)]$. Shortly after turning back, the vortex exhibits another complex reshaping process evidenced by a twist in its power curve [see the inset in Fig. 7 (b) near point ' $i$ ']. Afterwards, the power curve moves into the semi-infinite gap smoothly, and the vortex ultimately becomes four fundamental solitons with $\pi / 2$ phase delay between each other. Fig. 8 (l) shows such a vortex when the propagation constant $\mu=1$, which is far away from the Bloch band. The complexity of the power curve as well as the intricate reshaping process of vortices exhibited in this saturable-vortex family is very remarkable and is rarely seen in other wave systems.

Now we consider off-site vortex solitons under focusing saturable nonlinearity. Again, many families of them are found. One such family is shown in Fig. 9 (power curve) and Fig. 10 (vortex profiles). We see that this power curve as well as its vortex reshaping process are simpler. Indeed, unlike the above on-site saturable case, the power curve here has no twists. It does have a turning point near the Bloch band though, just like all other vortex families reported in this paper. At high powers, the off-site vortex has a ring profile [Fig. 10 (a)]. As its power decreases (with increasing $\mu$ values), it reshapes itself into a four-humped off-site vortex occupying four adjacent lattice sites [Fig. 10 (b, c)]. These vortices have been theoretically analyzed and experimentally observed in [21, 22, 36]. Comparing these vortices with those of off-site Kerr vortices in Fig. 3, we can see that this saturable-vortex family is the counterpart of the first off-site Kerr-vortex family. As the propagation constant $\mu$ moves from the lower branch to the upper one, this saturable vortex continuously reshapes itself, and eventually turns into four humps with $\pi / 2$ phase delay between each other (as in the on-site case). In all vortices of this off-site family, their phase fields have simple $2 \pi$-winding around the vortex center [see inset in Fig. 10 (a)], thus having charge one.

When the saturable nonlinearity is of defocusing type $\left(E_{0}<0\right)$, infinite families of vortex solitons exist in the first band gap. In this case, we have found that saturable vortices show similar behaviors (such as power curves and vortex shapes) as defocusing Kerr vortices in Figs. 446, thus such results will not be displayed here.

\section{SUMMARY AND DISCUSSION}

In this paper, we examined various families of chargeone vortex solitons in a $2 \mathrm{D}$ periodic media. For both Kerr and saturable nonlinearities, we found that there exist infinite families of on-site and off-site vortex solitons in the semi-infinite gap (for focusing nonlinearity) and the first gap (for defocusing nonlinearity). We further showed that all these vortex families do not bifurcate from edges of Bloch bands. Rather, before reaching band edges, they turn back and move into band gaps again. Within each vortex family, we revealed that topological structures of vortex solitons undergo drastic changes (such as evolving from a square configuration to a cross configuration) as the propagation constant varies.

Regarding the linear stability of vortex solitons, it has been shown before that certain on-site and off-site chargeone vortex solitons in the semi-infinite gap (under focusing nonlinearity) are stable in deep lattice potentials or 
(a)

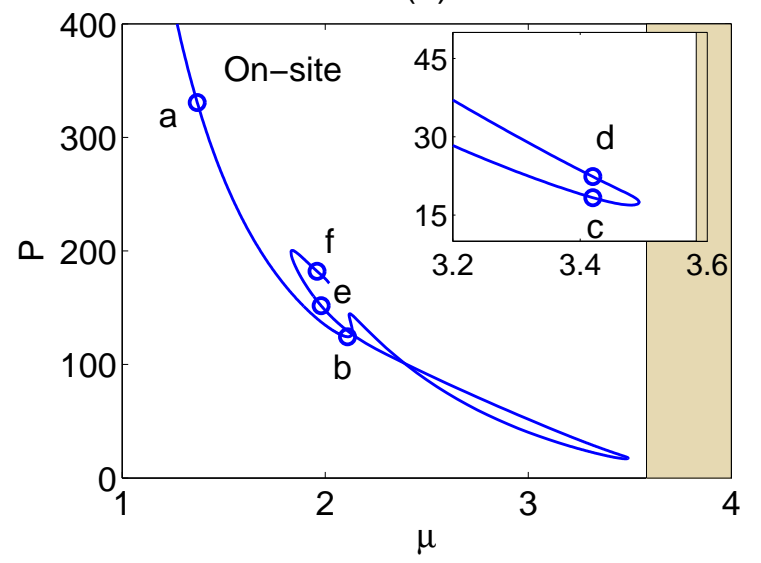

(b)

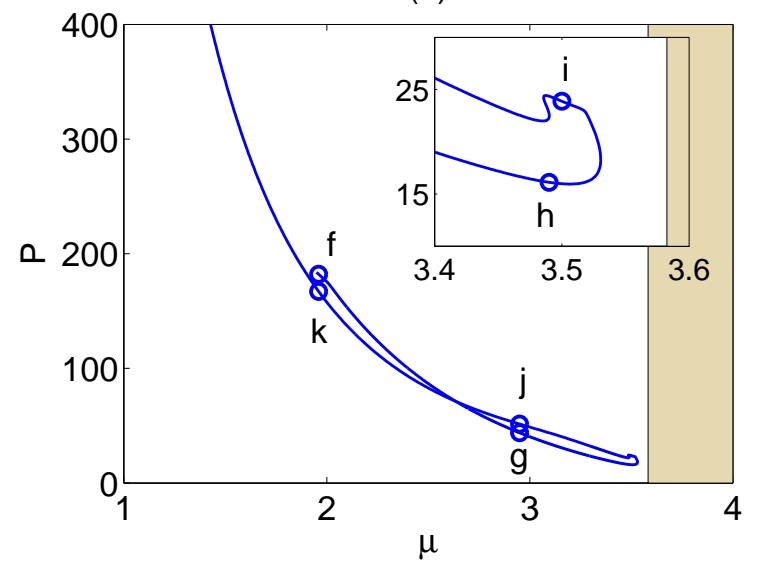

FIG. 7: Power diagrams of an on-site vortex family in the semi-infinite gap under focusing saturable nonlinearity. The power curve after point ' $\mathrm{f}$ ' in (a) is shown in (b). Vortex profiles at the marked points are shown in Fig. 8 . The insets zoom in on the graphs near the band edge.

(a)

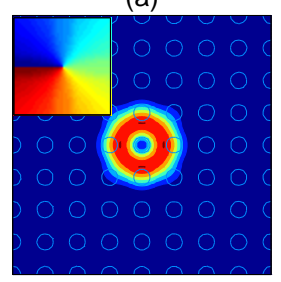

(g)

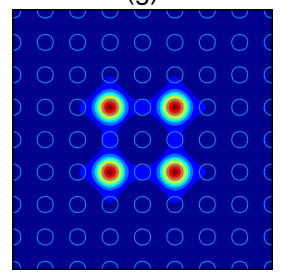

(b)

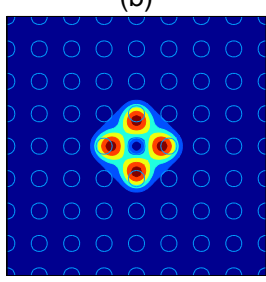

(h)

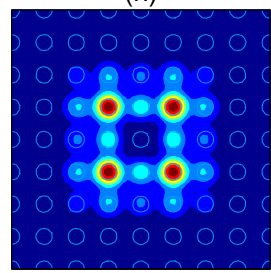

(c)

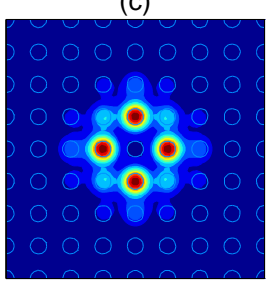

(i)

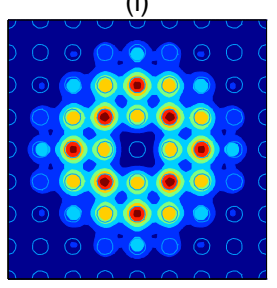

(d)

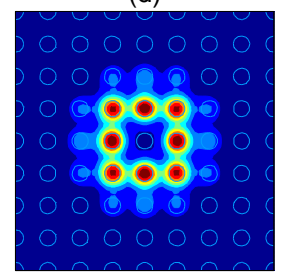

(j)

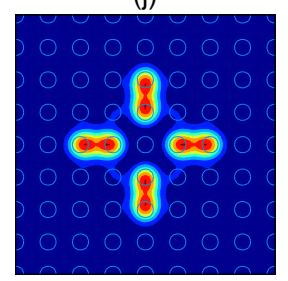

(e)

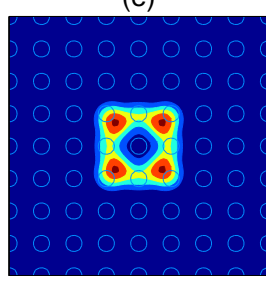

(k)

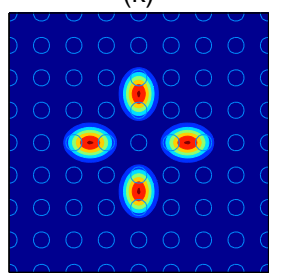

(f)

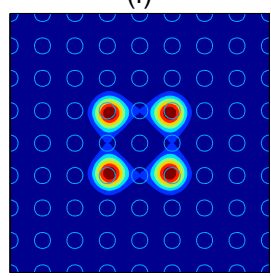

(l)

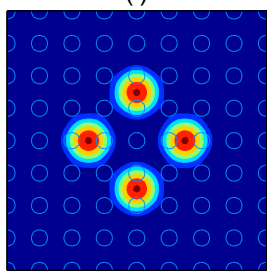

FIG. 8: (Color online) On-site vortex profiles $(|u|)$ corresponding to the marked points in Fig. [7 under focusing saturable nonlinearity. (l) shows the profile of the vortex at $\mu=1$ (far from the band edge). The inset in (a) is the typical phase structure of all these vortices. The background circles represent the lattice sites (with high $I$ values), as in Fig. 10.

with weak intersite couplings [13, 20, 27, 36, 40]. Certain on-site and off-site gap vortices under defocusing nonlinearity are stable as well [25, 27]. Such vortices generally correspond to the lower branches of first-family vortices reported in this paper [see Figs. 3(a) and 6(b) for instance]. It will be interesting to investigate the linear stability of upper branches of first-family vortices as well as branches of higher-family vortices obtained in this paper. The stability results in [40] imply that some of the upper branches of vortices are linearly unstable [such as Fig. 2(d)]. In such cases, one can ask where on the vortex branches the instability starts to appear. Note that all vortices reported in this paper have charge one. It is known that vortices with more intensity humps can be stabilized by increasing their charges [40, 41]. For instance, the vortex in Fig. 2(d) can be stabilized if its charge increases to three [40]. Thus it would be interesting to study vortex families with higher charges as well as their stability properties. These questions will be left for future studies.

\section{ACKNOWLEDGMENTS}

This work was supported in part by the Air Force Office of Scientific Research under Grant No. USAF 955005-1-0379. 


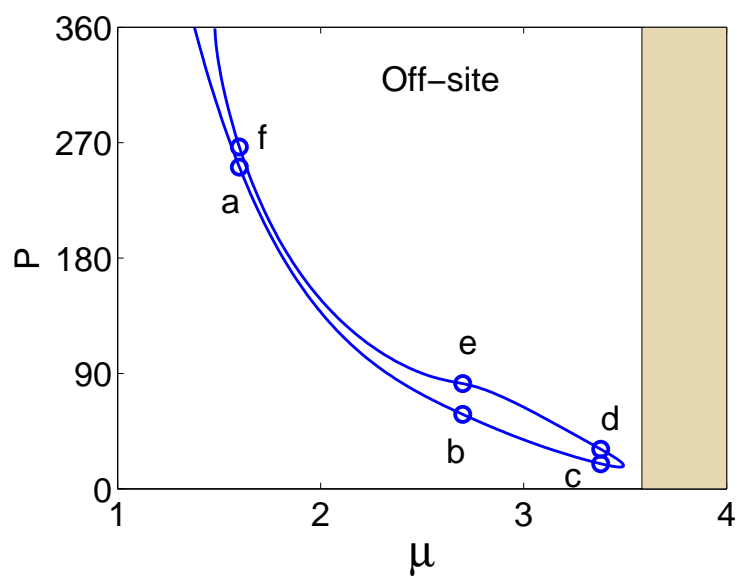

FIG. 9: The power diagram of an off-site vortex family in the semi-infinite gap under focusing saturable nonlinearity. Vortex profiles at the marked points are shown in Fig. 10.

(a)

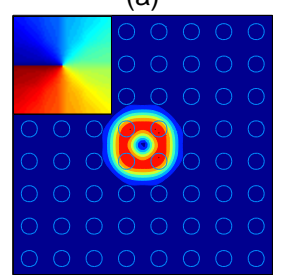

(d)

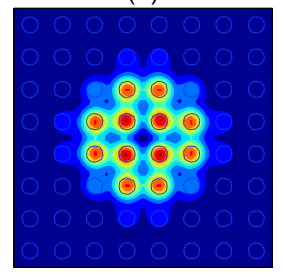

(b)

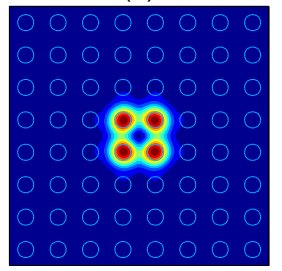

(e)

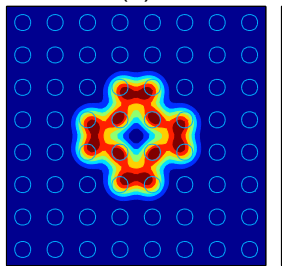

(c)

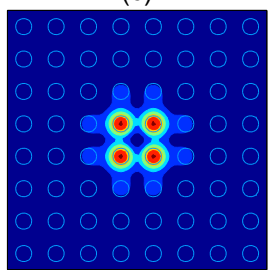

(f)

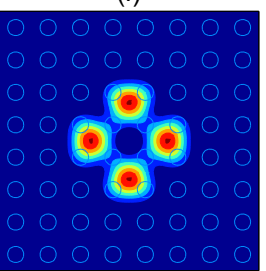

FIG. 10: (Color online) Off-site vortex profiles $(|u|)$ corresponding to the marked points in Fig. 9 under focusing saturable nonlinearity. The inset in (a) is the typical phase structure of all these vortices.

[1] J. D. Joannopoulos, R. D. Meade, and J. N. Winn, Photonic Crystals: Molding the Flow of Light, Princeton University Press, Princeton, NJ, 1995.

[2] P. Russell, "Photonic crystal fibers", Science 299, 358 (2003).

[3] D. N. Christodoulides, F. Lederer, and Y. Silberberg, "Discretizing light behaviour in linear and nonlinear waveguide lattices", Nature (London) 424, 817 (2003).

[4] D. K. Campbell, S. Flach, and Yu. S. Kivshar, "Localizing energy through nonlinearity and discreteness ", Phys. Today 57, 43-49 (2004).

[5] F. Dalfovo, S. Giorgini, L. P. Pitaevskii and S. Stringari, "Theory of Bose-Einstein condensation in trapped gases", Rev. Mod. Phys. 71, 463 (1999).

[6] Th. Anker, M. Albiez, R. Gati, S. Hunsmann, B. Eier- mann, A. Trombettoni, and M. K. Oberthaler, "Nonlinear Self-Trapping of Matter Waves in Periodic Potentials", Phys. Rev. Lett. 94, 020403(2005).

[7] K. Xu, Y. Liu, J.R. Abo-Shaeer, T. Mukaiyama, J.K. Chin, D.E. Miller, W. Ketterle, K.M. Jones, and E. Tiesinga, "Sodium Bose-Einstein Condensates in an Optical Lattice", Phys. Rev. A 72, 043604 (2005).

[8] Y. S. Kivshar, "Self-localization in arrays of defocusing waveguides", Opt. Lett. 18, 1147 (1993).

[9] J. W. Fleischer, Mordechai Segev, Nikolaos K. Efrimidis and D. N. Christodoulides, "Observation of twodimensional discrete solitons in optically induced nonlinear photonic lattices", Nature 422, 147 (2003).

[10] C. Lou, X. Wang, J. Xu, Z. Chen, and J. Yang, "Nonlinear spectrum reshaping and gap-soliton-train trapping in 
optically induced photonic structures", Phys. Rev. Lett. 98, 213903 (2007).

[11] D. N. Christodoulides and R.I. Joseph, "Discrete selffocusing in nonlinear arrays of coupled waveguides", Opt. Lett. 13, 794 (1988).

[12] H.S. Eisenberg et al., "Discrete Spatial Optical Solitons in Waveguide Arrays", Phys. Rev. Lett. 81, 3383 (1998).

[13] J. Yang and Z. H. Musslimani, "Fundamental and vortex solitons in a two-dimensional optical lattice", Opt. Lett. 28, 2094 (2003).

[14] N. K. Efremidis, J. Hudock, D. N. Christodoulides, J. W. Fleischer, O. Cohen, and M. Segev, "Two-Dimensional Optical Lattice Solitons ", Phys. Rev. Lett. 91, 213906 (2003).

[15] D. E. Pelinovsky, A. A. Sukhorukov, and Y. S. Kivshar, "Bifurcations and stability of gap solitons in periodic potentials", Phys. Rev. E 70, 036618 (2004).

[16] H. Martin, E. D. Eugenieva, Z. Chen, and D. N. Christodoulides, "Discrete Solitons and Soliton-Induced Dislocations in Partially Coherent Photonic Lattices", Phys. Rev. Lett. 92, 123902 (2004).

[17] R. Iwanow, R. Schiek, G. I. Stegeman, T. Pertsch, F. Lederer, Y. Min, and W. Sohler, "Observation of Discrete Quadratic Solitons", Phys. Rev. Lett. 93, 113902 (2004).

[18] D. Neshev, E. Ostrovskaya, Yu. S. Kivshar, and W. Krolikowski, "Spatial solitons in optically induced gratings", Opt. Lett. 28, 710 (2003).

[19] J. Yang, I. Makasyuk, A. Bezryadina, and Z. Chen, "Dipole and quadrupole solitons in optically-induced two-dimensional photonic lattices: theory and experiment", Stud. Appl. Math. 113, 389 (2004).

[20] B. A. Malomed and P. G. Kevrekidis, "Discrete vortex solitons ", Phys. Rev. E 64, 026601 (2001).

[21] D. N. Neshev, T. J. Alexander, E. A. Ostrovskaya, Y. S. Kivshar, H. Martin, I. Makasyuk, and Z. Chen, "Observation of Discrete Vortex Solitons in Optically Induced Photonic Lattices", Phys. Rev. Lett. 92, 123903 (2004).

[22] J. W. Fleischer, G. Bartal, O. Cohen, O. Manela, M. Segev, J. Hudock, and D. N. Christodoulides, "Observation of Vortex-Ring "Discrete" Solitons in 2D Photonic Lattices", Phys. Rev. Lett. 92, 123904 (2004).

[23] T.J. Alexander, A.A. Sukhorukov, Yu.S. Kivshar, "Asymmetric vortex solitons in nonlinear periodic lattices", Phys. Rev. Lett. 93063901 (2004).

[24] R. Fischer, D. Trager, D. N. Neshev, A. A. Sukhorukov, W. Krolikowski, C. Denz, and Y. S. Kivshar, "ReducedSymmetry Two-Dimensional Solitons in Photonic Lattices", Phys. Rev. Lett. 96, 023905 (2006).

[25] Elena A. Ostrovskaya and Yuris S. Kivshar, "MatterWave Gap Vortices in Optical Lattices", Phys. Rev. Lett.
93, 160405 (2004).

[26] G. Bartal, O. Manela, O. Cohen, J. W. Fleischer and M. Segev, "Observation of second-band vortex solitons in 2D photonic lattices", Phys. Rev. Lett. 95, 053904 (2005).

[27] T. Richter and F. Kaiser, "Anisotropic gap vortices in photorefractive media", Phys. Rev. A 76, 033818 (2007).

[28] X. Wang, Z. Chen, J. Wang and J. Yang, "Observation of in-band lattice solitons", to appear in Phys. Rev. Lett.

[29] Y. V. Kartashov, V. A. Vysloukh, and L. Torner, "Rotary Solitons in Bessel Optical Lattices", Phys. Rev. Lett. 93, 093904 (2004).

[30] X. Wang, Z. Chen, and P. G. Kevrekidis, "Observation of Discrete Solitons and Soliton Rotation in Optically Induced Periodic Ring Lattices", Phys. Rev. Lett. 96, 083904 (2006).

[31] Z. Shi and J. Yang, "Solitary waves bifurcated from Bloch bands in two-dimensional periodic media", Phys. Rev. E 75, 056602 (2007).

[32] Z. Musslimani and J. Yang, "Self-trapping of light in a two-dimensional periodic structure" J. Opt. Soc. Am. B. 21, 973-981 (2004)

[33] Elena A. Ostrovskaya, Tristram J. Alexander and Yuris S. Kivshar, "Generation and detection of matter-wave gap vortices in opticall lattices.", Phys. Rev. A 74, 023605 (2006).

[34] A. Szameit, J. Burghoff, T. Pertsch, S. Nolte, A. Tnnermann, and F. Lederer, "Two-dimensional soliton in cubic fs laser written waveguide arrays in fused silica," Opt. Express 14, 6055-6062 (2006).

[35] J. Yang and T. I. Lakoba, "Squared-operator iteration methods for solitary waves in general nonlinear wave equation.", Stud. Appl. Math. 118, 153 (2007).

[36] J. Yang, "Stability of vortex solitons in a photorefractive optical lattice", New J. Phys. 6, 47 (2004).

[37] T. S. Yang and T. R. Akylas, "On asymmetric gravity capillary solitary waves", J. Fluid Mech. 330, 215 (1997).

[38] N.K. Efremidis, S. Sears, D.N. Christodoulides, J.W. Fleischer, and M. Segev, "Discrete solitons in photorefractive optically induced photonic lattices", Phys. Rev. E 66, 046602 (2002).

[39] J. Wang, F. Ye, L. Dong, T. Cai and Y. Li, "Lattice solitons suported by competing cubic-quintic nonlinearity.", Phys. Letts. A, 339, 74 (2005).

[40] D.E. Pelinovsky, P.G. Kevrekidis and D.J. Frantzeskakis, "Persistence and stability of discrete vortices in nonlinear Schrodinger lattices", Physica D 212, 20-53 (2005).

[41] M. Oster and M. Johansson, "Stable stationary and quasiperiodic discrete vortex breathers with topological charge S=2", Phys. Rev. E 73, 066608 (2006). 